\section{Rastreamento mamográfico do câncer de mama no Sul do Brasil e fatores associados: estudo de base populacional}

\author{
Mammogram screening for breast cancer and \\ associated factors in the South of Brazil: \\ a based-population survey \\ El cribado mamográfico para el cáncer de mama \\ en el sur de Brasil y factores asociados: \\ un estudio basado en la población
}

\begin{abstract}
The purpose of this study was to identify factors associated with annual mammogram screening in women 40 to 69 years of age in Florianópolis, Santa Catarina State, Brazil. Data were obtained from two population studies with independent samples, one with adult women and the other with elderly women, in 2009-2010. Prevalence rates for annual mammogram screening were estimated by crude and adjusted Poisson regression. Overall prevalence of annual mammograms in adult women was 43.5\% (95\% CI: 38.848.2) and was higher in women with private health insurance. In elderly women, prevalence was 38.3\% (95\%CI: 34.0-42.6), with the following associated factors: marital status (married/ with partner), schooling (> 5 years), and highest income quartile. Mammogram screening should be independent of socioeconomic status and age in order to reduce mortality from breast cancer.
\end{abstract}

Early Diagnosis; Mammography; Breast Neoplasms; Health Surveys
Ione Jayce Ceola Schneider 1,2 Marui Weber Corseuil Giehl 1 Antonio Fernando Boing 1 Eleonora d'Orsi 1

\section{Resumo}

O objetivo foi identificar os fatores associados à realização anual de mamografia em mulheres de 40 a 69 anos residentes em Florianópolis, Santa Catarina, Brasil. Os dados foram obtidos em dois inquéritos populacionais, um com adultas e outro com idosas, em 2009-2010. Foi estimada a prevalência de realização anual de mamografia e se empregou regressão univariada e multivariada de Poisson para identificar os fatores associados. Participaram do estudo 447 adultas e 510 idosas. A prevalência de realização anual entre adultas foi 43,5\% (IC95\%: 38,8-48,2), e possuir plano de saúde privado aumentou esta prevalência. Para as idosas, 38,3\% (IC95\%: 34,0-42,6) realizaram mamografia anual e os fatores associados foram ter companheiro, mais de cinco anos de estudo e pertencer ao quartil de renda mais alto. A utilização do exame de mamografia deve ser independente de qualquer condicionante social, em qualquer faixa etária, promovendo assim redução da mortalidade por câncer de mama.

Diagnóstico Precoce; Mamografia; Neoplasias de Mama; Inquéritos Epidemiológicos 


\section{Introdução}

A mamografia é usada como ferramenta diagnóstica para examinar pacientes sintomáticas ou como técnica de rastreamento para o câncer de mama 1,2. É efetiva para diagnóstico precoce de doença invasiva que pode levar de 5 a 7 anos para progredir 1 , podendo detectar $80-90 \%$ dos casos de câncer de mama em mulheres assintomáticas ${ }^{3}$. A Organização Mundial da Saúde (OMS) reconhece a mamografia como método para rastreamento do câncer de mama por reduzir o diagnóstico em estágio avançado, aumentar a sobrevida e diminuir a mortalidade 4 .

O desempenho da mamografia de rastreamento é diferente segundo as faixas etárias, mas em todas há evidência de redução da mortalidade. Em mulheres de 40 a 49 anos, se o rastreamento for realizado a cada dois anos, pode ocorrer redução de até $15 \%$ na mortalidade por câncer de mama, porém nestas mulheres pode haver alta taxa de falsos positivos, com necessidade de exames adicionais ${ }^{5}$. Redução semelhante na mortalidade é encontrada em mulheres de 50 a 59 anos, assim como taxa de falsos positivos 5 . E o melhor desempenho é encontrado na faixa etária de 60 a 69 anos, com maior redução da mortalidade, $32 \%$, e da taxa de falsos positivos 5 .

A frequência com que as mulheres são rastreadas é uma decisão que deve ser planejada. Este intervalo depende da história natural da doença, e também dos recursos disponíveis para o programa ${ }^{4}$. Existem evidências de que o rastreamento bienal mantém a redução da mortalidade 5,6. Mas existem entidades científicas, como a American Cancer Society 3,7,8 que defendem a recomendação anual a partir dos 40 anos. Esta entidade aponta, entre os benefícios da mamografia anual, a redução do risco de morte por câncer de mama, assim como cirurgias e terapias adjuvantes menos agressivas, devido ao diagnóstico precoce das lesões.

A política pública brasileira segue as recomendações do Instituto Nacional de Câncer (INCA). Este recomenda que rastreamento do câncer de mama por mamografia seja realizado em mulheres entre 50 e 69 anos, com no máximo dois anos de intervalo 9,10. Entretanto, o rastreamento é de forma oportunística 11, no qual a solicitação do exame dependerá do interesse da mulher em procurar o serviço de saúde 12,13. Além disto, não há evidência de que o programa de controle do câncer de mama seja efetivo ${ }^{11}$, pois não ocorre acompanhamento dos casos suspeitos, premissas nos rastreamentos organizados 12,13 .

Contudo, a Sociedade Brasileira de Mastologia 14 e a Comissão Nacional de Qualidade em
Mamografia do Colégio Brasileiro de Radiologia e Diagnóstico por Imagem 15, seguem as recomendações de algumas entidades internacionais quanto à realização anual da mamografia a partir dos 40 anos, devido aos benefícios descritos acima.

Apesar da recomendação do INCA 9,10 para realização da mamografia acima dos 50 anos, a análise do Suplemento de Saúde da Pesquisa Nacional por Amostra de Domicílios de 2008 revelou que $67 \%$ das mulheres de 40 a 49 anos já realizaram o exame, percentual semelhante àquelas de 50 a 59 anos $(71,5 \%) 16$.

Estudos nacionais revelam que a realização de mamografia, conforme recomenda o INCA 9,10 , não acontece de forma igualitária. Mulheres de 50 a 69 anos 16,17, de cor/raça branca 17, com maior nível de escolaridade $16,18,19$, maior renda $16,17,20,21,22$, que residem com companheiro 16,19 , com plano de saúde privado $16,19,23,24$, que tenham realizado exame clínico das mamas $22 \mathrm{e}$ frequentado consulta ginecológica 19,22 apresentam maior prevalência de realização do exame.

No Brasil, dados dos Registros de Câncer de Base Populacional brasileiros de 1993 a 2006 mostram que as taxas de incidência de câncer de mama em mulheres de 40 a 49 anos são semelhantes àquelas de 50 anos ou mais 25 . Estudo realizado na região de Barretos, São Paulo, mostrou que a chance de diagnóstico de câncer de mama, em mulheres de 45 a 49 anos, é equivalente àquelas de 60 a 69 anos 26. Parte do aumento da taxa de incidência nesta população pode ser devido aos programas de rastreamento por mamografia detectando lesões iniciais 27 .

O câncer de mama, na maioria das vezes, é um câncer de bom prognóstico se diagnosticado precocemente. Assim, o objetivo do presente artigo é identificar os fatores associados à realização anual de mamografia e descrever as barreiras para realização desta em mulheres de 40 a 69 anos, residentes em Florianópolis, Santa Catarina.

\section{Métodos}

No Município de Florianópolis, capital do Estado de Santa Catarina, ocorreram, simultaneamente e de maneira independente, nos anos de 2009 e 2010, dois inquéritos populacionais. Um deles foi realizado com a população adulta (20 a 59 anos) e outro com a população idosa (60 anos ou mais), denominados EpiFloripa Adulto e EpiFloripa Idoso, respectivamente. Estes inquéritos tiveram como objetivo geral estudar as condições de saúde da população residente. Em 2009, a população estimada do município foi de 408.163 habitan- 
tes, com aproximadamente $60 \%$ adultos $(51,7 \%$ mulheres) e $10,8 \%$ idosos (57\% mulheres). A cidade de Florianópolis, no ano de 2000, ocupava a sexta posição do Índice de Desenvolvimento Municipal (IDH-M), com índice de 0,766, e em 2010 apresentou índice de 0,847 , ocupando a terceira posição entre os municípios brasileiros e a primeira posição entre as capitais (Programa das Nações Unidas para o Desenvolvimento. Atlas do Desenvolvimento Humano no Brasil 2013. Ranking - Todo o Brasil (2010). http:/ / atlasbrasil. org.br/2013/ranking, acessado em 08/Set/2013).

No estudo de realização anual de mamografia e fatores associados foram selecionadas somente as mulheres adultas de 40 a 59 anos e as idosas de 60 a 69 anos. O tamanho da amostra necessário para cada estudo foi calculado de forma independente com o auxílio do programa Open Epi Version 2 (Dean AG, Sullivan KM, Soe MM. OpenEpi: Open Source Epidemiologic Statistics for Public Health, http://www.OpenEpi.com). Consideraram-se como parâmetros prevalência estimada de 50\%, nível de 95\% de confiança e erro amostral igual a 7 pontos percentuais para adultas e 6,5 para idosas, efeito de delineamento de 2, tamanho da população de adultas de 55.003 e de idosas de 14.056. Por fim, no inquérito com as adultas foram acrescidos $10 \%$ para compensar as perdas previstas e $20 \%$ para estudo de associações; e nas idosas $15 \%$ para perdas e $20 \%$ para os estudos de associações. Assim, definiram-se como amostra mínima 508 adultas e 560 idosas.

Foi possível calcular a posteriori o poder estatístico da amostra. A amostra de adultas permitiu identificar uma razão de prevalência de 1,4 , considerando poder de $80 \%$, erro alfa de $5 \%$, prevalência nos expostos de 58,1\% e nos não expostos de $42,4 \%$. Para as idosas, considerando a prevalência de $64,2 \%$ nos expostos e $29,2 \%$ nos não expostos, permitiu identificar uma razão de prevalência de 2,2.

O processo de seleção da amostra deu-se por conglomerados em dois estágios. No primeiro sortearam-se sistematicamente os setores censitários, estratificados em decis segundo a renda média mensal do chefe da família, e no segundo, os domicílios. Realizou-se atualização do número de domicílios particulares permanentes ocupados nos setores sorteados, pois o último censo do Instituto Brasileiro de Geografia e Estatística (IBGE) havia sido realizado em 2000.

No EpiFloripa Adulto foram sorteados 60 setores censitários (seis por decil de renda), enquanto no EpiFloripa Idoso foram sorteados 80 setores censitários (oito por decil de renda). Para reduzir o coeficiente de variação do número de domicílios por setor censitário, foi realizado o agrupamento de unidades com menos de 150 domicílios e divisão daqueles com mais de 500 domicílios, respeitando-se o decil de renda correspondente e a proximidade geográfica. Esse procedimento resultou em 63 setores no estudo com adultos (coeficiente de variação de $32 \%$ ) e 83 setores no estudo do idoso (coeficiente de variação de $35,2 \%$ ). Em seguida foram sorteados sistematicamente, em cada setor, 18 domicílios no estudo com adultos e 64 domicílios no estudo com idosos, considerando-se a média de 1,8 residentes adultos por domicílio e 0,34 residente idoso.

Todas as residentes dos domicílios sorteados, na faixa etária de cada estudo, foram convidadas a participar. Foram consideradas perdas as pessoas cujo domicílio foi visitado ao menos quatro vezes sem localizá-las, sendo no mínimo uma visita no fim de semana e outra no período noturno. Foram excluídas da análise as mulheres que relataram diagnóstico prévio de câncer de mama ( $n=12$ entre idosas e $n=13$ entre adultas) e entrevistas respondidas por informantes $(n=4$ entre idosas). Houve verificação semanal da consistência dos dados e controle de qualidade por meio de aplicação por telefone de um questionário reduzido, em aproximadamente $15 \%$ das entrevistas dos adultos e $10 \%$ dos idosos, selecionadas aleatoriamente.

No presente estudo utilizou-se somente o conceito "prática" da metodologia CAP (Conhecimento, Atitude e Prática) caracterizado como a tomada de decisão para a realização da mamografia 20,21. Também foram incluídas as informações sobre barreiras para sua realização e os motivos que levam os médicos a solicitarem o exame.

A variável dependente foi definida através da utilização das respostas das questões " $A$ senhora já fez mamografia alguma vez na vida?”, “Quanto tempo faz que a senhora fez a última mamografia?" e "De quanto em quanto tempo a senhora tem feitos os exames de mamografia?". Considerou-se a realização anual aquelas que realizaram mamografia no último ano, com intervalo máximo de um ano, conforme recomendação da American Cancer Society 8 , Sociedade Brasileira de Mastologia 14 e Colégio Brasileiro de Radiologia e Diagnóstico por Imagem 15 para diagnóstico precoce do câncer de mama.

As variáveis independentes analisadas foram estado civil (viver com companheiro ou viver sem companheiro), renda familiar per capita (em quartis de renda), escolaridade em anos de estudo (0 a 4, 5 a 8, 9 a 11 e 12 ou mais, para adultas; 0,1 a 4,5 a 8,9 a 11, 12 ou mais para idosas), cor da pele autorreferida [(branca, preta ou negra e parda); amarelas ( $n=7$ entre adultas e $n=3$ entre idosas) e indígenas ( $n=5$ entre adultas e $n=4$ en- 
tre idosas) foram excluídas da análise por apresentar baixa amostra)], trabalho remunerado no último mês (sim, não), posse de plano de saúde privado (sim, não). Foram excluídas as mulheres com diagnóstico de câncer de mama ( $\mathrm{n}=12$ idosas e $\mathrm{n}=13$ adultas) e as entrevistas respondidas por informantes ( $\mathrm{n}=4$ idosas).

Além disto, foram investigados, através de pergunta fechada, os motivos que levaram as mulheres a fazerem a mamografia (exame de rotina ou acompanhamento, suspeita ou nódulo na mama, familiar ou amigo com câncer de mama, medo de ter câncer, conhecimento de que mulher acima de 40 anos deve realizar o exame) ou a não realizarem o exame (medo de diagnosticar um câncer, achar desnecessário, falta de solicitação médica).

A análise estatística foi realizada de forma separada para adultas (40 a 59 anos) e idosas (60 anos a 69 anos) e conduzida no programa Stata/SE 9.0 (StataCorp., College Station, Estados Unidos) considerando-se a amostra complexa e incorporando-se os pesos amostrais. Utilizou-se análise descritiva das variáveis e análise bivariada para calcular as prevalências do desfecho, para as variáveis categóricas, e medidas de tendência central para as contínuas. Em seguida realizou-se análise bruta e multivariada através da regressão de Poisson para estimar razões de prevalências e respectivos intervalos de $95 \%$ de confiança (IC95\%). Obtiveram-se os valores de $\mathrm{p}$ por meio do teste de Wald 28 . Foram incluídas na análise ajustada as variáveis que apresentaram valor de $\mathrm{p}<0,20$ na análise bruta, sendo incluídas uma a uma no modelo. Permaneceram no modelo final as variáveis com valor de $\mathrm{p}<0,05$ e/ou que ajustassem a análise.

Os inquéritos foram aprovados pelo Comitê de Ética em Pesquisa da Universidade Federal de Santa Catarina (UFSC), sob protocolos de ㄲo $351 / 2008$ e 352/2008, adulto e idoso, respectivamente. Os pesquisadores declaram não haver conflito de interesses.

\section{Resultados}

Das adultas participantes do EpiFloripa Adulto fizeram parte deste estudo 447 mulheres (taxa de resposta de $88 \%$ ). Destas, $56,6 \%$ tinham de 40 a 49 anos, $35,8 \%$ tinham nível superior e a maioria declarou cor da pele branca $(87,6 \%)$, vivia com companheiro (68\%), estava trabalhando $(62,2 \%)$ e possuía plano de saúde privado $(65,6 \%)$ (Tabela 1$)$.

A prevalência de realização anual de mamografia neste grupo de mulheres foi de $43,5 \%$ (IC95\%: 38,9-48,2). Na análise bivariada os fato- res associados à realização anual foram escolaridade maior de nove anos, aumento da renda, plano de saúde privado. Permaneceu na análise ajustada, por faixa etária, somente possuir plano de saúde privado. Mulheres que possuíam plano de saúde privado apresentaram prevalência $50 \%$ maior comparadas às que não possuíam (Tabela 1).

Das mulheres de 60 a 69 anos participantes do EpiFloripa Idoso, 510 (taxa de resposta de $91,1 \%)$ participaram desta pesquisa. Destas, $86,7 \%$ declararam a cor da pele como branca, a maioria vivia com companheiro $(57,3 \%)$, tinha até oito anos de estudo (60,3\%), não tinha trabalho atual $(83,9 \%)$ e não possuía plano de saúde privado $(64,7 \%)$ (Tabela 2$)$.

A prevalência de realização anual de mamografia em mulheres de 60 a 69 anos foi de 38,3\% (IC95\%: 34,0-42,6). Na análise bivariada os fatores associados ao desfecho foram: cor da pele parda, possuir companheiro, não ser analfabeta, maior renda e possuir plano de saúde privado. Permaneceram na análise ajustada possuir companheiro, ter mais de cinco anos de escolaridade e pertencer ao quartil de renda mais alto. Em relação ao companheiro, as mulheres que se declararam ter companheiro apresentaram prevalência $47 \%$ maior de realização anual de mamografia. O aumento da escolaridade aumentou a realização anual de mamografia, mulheres com 12 anos ou mais de estudo apresentaram prevalência quatro vezes maior de realização em relação às analfabetas. Pertencer ao quarto quartil de renda (mais ricas) aumentou aproximadamente em duas vezes a prevalência em relação às do primeiro quartil (Tabela 2).

Das adultas participantes, 89,9\% já realizaram mamografia alguma vez na vida. Entre os motivos que as levaram a realizar o exame os mais citados foram rotina ou acompanhamento (75,4\%) e nódulo ou suspeita de nódulo (15,2\%). Para as mulheres que nunca realizaram o exame, a maioria foi por falta de solicitação $(51,2 \%)$ - destas, 80\% tinham entre 40 e 49 anos -, seguido de medo ou vergonha do exame $(30,2 \%)$ (Tabela 3).

Das idosas participantes, 90,7\% realizaram mamografia alguma vez na vida. Entre os motivos que as levaram a realizar o exame os mais citados foram rotina ou acompanhamento $(79,3 \%)$ e nódulo ou suspeita de nódulo (9,9\%). Para as mulheres que nunca realizaram, a maioria foi por falta de solicitação $(52,6 \%)$ e achar o exame desnecessário (42,1\%) (Tabela 3). 
Análise descritiva, bivariada e razão de prevalência bruta e ajustada para realização anual de mamografia, mulheres de 40 a 59 anos. EpiFloripa Adulto, Florianópolis, Santa Catarina, Brasil, 2009.

\begin{tabular}{|c|c|c|c|c|c|}
\hline Variáveis & n (\%) & $\begin{array}{c}\text { Realização } \\
\text { anual (\%) }\end{array}$ & Valor de $p$ & $\begin{array}{l}\text { RP bruta } \\
\text { (IC95\%) }\end{array}$ & $\begin{array}{l}\text { RP ajustada } \\
\text { (IC95\%) * }\end{array}$ \\
\hline \multicolumn{6}{|c|}{ Faixa etária [anos] ( $n=447)$} \\
\hline $50-59$ & $194(43,4)$ & $88(46,3)$ & 0,306 & 1,00 & \\
\hline $40-49$ & $253(56,6)$ & $104(41,4)$ & & $0,87(0,71-1,05)$ & \\
\hline \multicolumn{6}{|l|}{ Cor da pele $(n=434)$} \\
\hline Branca & $380(87,6)$ & $164(43,7)$ & 0,386 & 1,00 & \\
\hline Parda & $31(7,1)$ & $15(48,4)$ & & $1,10(0,76-1,59)$ & \\
\hline Preta ou negra & $23(5,3)$ & $7(30,4)$ & & $0,73(0,36-1,48)$ & \\
\hline \multicolumn{6}{|l|}{ Estado civil ( $n=447)$} \\
\hline Com companheiro & $304(68,0)$ & $137(45,5)$ & 0,219 & 1,00 & \\
\hline Sem companheiro & $143(32,0)$ & $55(39,3)$ & & $0,87(0,64-1,18)$ & \\
\hline \multicolumn{6}{|c|}{ Escolaridade [anos] $(n=447)$} \\
\hline 12 ou mais & $160(35,8)$ & $81(50,6)$ & 0,001 & $1,97(1,20-3,24)$ & ** \\
\hline $9-11$ & $133(29,7)$ & $63(47,4)$ & & $1,80(1,08-3,01)$ & \\
\hline $5-8$ & $78(17,5)$ & $30(40,5)$ & & $1,54(0,89-2,66)$ & \\
\hline $0-4$ & $76(17,0)$ & $18(24,3)$ & & 1,00 & \\
\hline \multicolumn{6}{|c|}{ Renda [quartis] $(n=436)$} \\
\hline 4 & $117(26,8)$ & $60(51,3)$ & 0,016 & $1,79(1,19-2,68)$ & ** \\
\hline 3 & $111(25,5)$ & $31(48,2)$ & & $1,71(1,15-2,54)$ & \\
\hline 2 & $106(24,3)$ & $42(40,0)$ & & $1,59(1,01-2,50)$ & \\
\hline 1 & $102(23,4)$ & $53(31,3)$ & & 1,00 & \\
\hline \multicolumn{6}{|l|}{ Trabalho atual $(n=447)$} \\
\hline Sim & $278(62,2)$ & $121(44,2)$ & 0,735 & $1,06(0,80-1,41)$ & ** \\
\hline Não & $169(37,8)$ & $71(42,5)$ & & 1,00 & \\
\hline \multicolumn{6}{|c|}{ Plano de saúde $(n=445)$} \\
\hline Sim & $292(65,6)$ & $149(51,6)$ & $<0,001$ & $1,80(1,31-2,47)$ & $1,50(1,08-2,09)$ \\
\hline Não & $153(34,4)$ & $43(28,5)$ & & 1,00 & 1,00 \\
\hline
\end{tabular}

* Ajustado por faixa etária;

** Perderam significância ao serem incluídas no modelo ajustado.

\section{Discussão}

O estudo permitiu conhecer os fatores associados à realização anual de mamografia em mulheres de 40 a 69 anos, assim como os motivos pelos quais estas realizam ou não o exame. Para as adultas, a realização anual esteve associada somente a possuir plano de saúde privado. Quanto às idosas, possuir mais de cinco anos de estudo e pertencer ao quarto quartil de renda familiar per capita e possuir companheiro aumentaram a prevalência de realização anual de mamografia.

Apesar dos estudos apresentarem metodologias diferentes que limitam comparações, a prevalência de realização de mamografia encontrada foi semelhante a outros estudos nacionais 17,20 e um internacional 29. No Município de Campi- nas, São Paulo, um estudo de base populacional verificou que $50,8 \%$ das residentes de 40 anos ou mais não haviam realizado mamografia nos dois anos anteriores à pesquisa, e houve associação com a cor da pele preta ou parda e renda familiar per capita menor de cinco salários mínimos 17. Marinho et al. 20 investigaram a prática adequada de mamografia, considerando-se a realização em período inferior a dois anos, na mesma cidade já citada, porém em serviços públicos. Os autores 20 constataram a prática adequada do desfecho em $35,7 \%$, em mulheres acima de 40 anos; associado à mulher ser casada e ter escolaridade superior a cinco anos de estudo.

Nos Estados Unidos, a realização do exame foi superior ao encontrado no presente estudo. Entre as mulheres de 40 a 49 anos, $49 \%$ não rea- 
Análise descritiva, bivariada e razão de prevalência bruta e ajustada para realização anual de mamografia, mulheres de 60 a 69 anos. EpiFloripa Idoso, Florianópolis, Santa Catarina, Brasil, 2009-2010.

\begin{tabular}{|c|c|c|c|c|c|}
\hline Variáveis & n (\%) & $\begin{array}{l}\text { Realização } \\
\text { anual (\%) }\end{array}$ & Valor de $\mathrm{p}$ & $\begin{array}{l}\text { RP bruta } \\
\text { (IC95\%) }\end{array}$ & $\begin{array}{l}\text { RP ajustada } \\
\text { (IC95\%) }\end{array}$ \\
\hline \multicolumn{6}{|l|}{ Cor da pele $(n=503)$} \\
\hline Branca & $436(86,7)$ & $172(40,6)$ & \multirow{3}{*}{0,044} & 1,00 & * \\
\hline Parda & $48(9,5)$ & $10(21,7)$ & & $0,48(0,25-0,90)$ & \\
\hline Preta ou negra & $19(3,8)$ & $7(36,8)$ & & $0,88(0,44-1,77)$ & \\
\hline \multicolumn{6}{|l|}{ Estado civil $(n=510)$} \\
\hline Com companheiro & $292(57,3)$ & $124(43,8)$ & \multirow{2}{*}{0,004} & $1,48(1,0,8-2,02)$ & $1,47(1,15-1,89)$ \\
\hline Sem companheiro & $218(42,7)$ & $66(31,0)$ & & 1,00 & 1,00 \\
\hline \multicolumn{6}{|c|}{ Escolaridade [anos] $(n=507)$} \\
\hline 12 ou mais & $122(24,1)$ & $60(51,3)$ & \multirow{5}{*}{$<0,001$} & $5,69(1,80-17,98)$ & $4,18(1,30-13,44)$ \\
\hline $9-11$ & $79(15,6)$ & $42(53,8)$ & & $6,31(1,92-20,74)$ & $4,36(1,32-14,47)$ \\
\hline $5-8$ & $103(20,3)$ & $34(34,3)$ & & $4,54(1,40-14-68)$ & $3,83(1,16-12,63)$ \\
\hline $1-4$ & $175(34,5)$ & $50(29,1)$ & & $3,47(1,01-12,04)$ & $3,22(0,93-11,18)$ \\
\hline 0 & $28(5,5)$ & $3(10,7)$ & & 1,00 & 1,00 \\
\hline \multicolumn{6}{|l|}{ Renda [quartis] $(n=510)$} \\
\hline 4 & $113(22,1)$ & $65(59,6)$ & \multirow{4}{*}{$<0,001$} & $2,41(1,63-3,57)$ & $1,98(1,29-3,04)$ \\
\hline 3 & $127(24,9)$ & $54(43,2)$ & & $1,80(1,17-2,76)$ & $1,51(0,99-2,30)$ \\
\hline 2 & $137(26,9)$ & $40(30,3)$ & & $1,36(0,78-2,37)$ & $1,29(0,766-2,16)$ \\
\hline 1 & $133(26,1)$ & $31(23,8)$ & & 1,00 & 1,00 \\
\hline \multicolumn{6}{|l|}{ Trabalho atual $(n=510)$} \\
\hline Não & $428(83,9)$ & $158(37,9)$ & \multirow{2}{*}{0,661} & 1,00 & \\
\hline Sim & $82(16,1)$ & $32(40,5)$ & & $1,04(0,78-1,40)$ & \\
\hline \multicolumn{6}{|c|}{ Plano de saúde $(n=510)$} \\
\hline $\operatorname{Sim}$ & $330(64,7)$ & $146(45,3)$ & \multirow{2}{*}{$<0,001$} & $1,76(1,34-2,33)$ & \\
\hline Não & $180(35,3)$ & $44(25,3)$ & & 1,00 & * \\
\hline
\end{tabular}

* Perderam significância ao serem incluídas no modelo ajustado.

lizavam mamografia anualmente, entre as de 50 a 59 anos, esta taxa foi de $55 \%$, e naquelas com 60 ou mais, foi de $57 \%$, e a realização do exame não foi associada à escolaridade 29 .

Os dados de realização anual de mamografia para mulheres residentes em Florianópolis assemelham-se aos encontrados no Município de São Paulo, pelo Inquérito de Saúde no Município de São Paulo (ISA-Capital) e Vigilância de Fatores de Risco e Proteção para Doenças Crônicas por Inquérito Telefônico (VIGITEL) São Paulo. Os dados demonstram que entre as mulheres acima de 40 anos, mais de $50 \%$ realizaram a mamografia no ano anterior à pesquisa e que as de 40 a 59 anos apresentaram maior prevalência comparadas às de 60 anos ou mais 30 .

No Brasil, dados do Sistema de Informação para o Controle do Câncer de Mama (SISMAMA), mostram que o número de mamografia triplicou em 2010 em relação a 2009. Entre as mulheres que realizaram o exame nos anos da pesquisa, mais de $86 \%$ tinham de 40 a 69 anos de idade 31 . Oliveira et al. 16 encontraram aumento da realização de mamografia no Brasil anterior ao período investigado (2003 a 2008). Na Região Sul, o aumento foi em torno de $15 \%$. O aumento da realização de mamografia também foi encontrado nas mulheres de 40 a 49 anos, semelhante às de 50 a 69 anos, e os autores afirmam que isto pode estar associado às recomendações de rastreamento das sociedades médicas internacionais, além da necessidade de acompanhamento de lesões 16.

Ao ser investigada a cobertura mamográfica em um programa de controle do câncer de mama no interior da Paraíba ${ }^{32}$, com os parâmetros de realização de mamografia de acordo com o Ministério da Saúde ${ }^{9}$, foi encontrado que entre as mulheres de 40 a 49 anos, 53,8\% haviam realizado mamografia, associado ou não a exame 
Análise descritiva dos motivos e barreiras para realização de mamografia, mulheres adultas e idosas. EpiFloripa Adulto e Epi

Floripa Idoso, Florianópolis, Santa Catarina, Brasil, 2009-1200.

\begin{tabular}{|c|c|c|c|c|}
\hline \multirow[t]{2}{*}{ Variáveis } & \multicolumn{2}{|c|}{ Adultas } & \multicolumn{2}{|c|}{ Idosas } \\
\hline & $\mathbf{n}$ & $\%($ IC95\%) & $\mathbf{n}$ & $\%($ IC95\%) \\
\hline \multicolumn{5}{|l|}{ Realizou mamografia alguma vez na vida } \\
\hline Sim & 396 & $89,9(86,9-92,6)$ & 439 & $90,7(88,1-93,3)$ \\
\hline Não & 45 & $10,2(7,4-13,0)$ & 45 & $9,3(6,7-11,9)$ \\
\hline Total & 441 & 100,0 & 484 & 100,0 \\
\hline \multicolumn{5}{|l|}{ Motivos para fazer a mamografia } \\
\hline Nódulo ou suspeita & 60 & $15,2(11,6-18,8)$ & 44 & $9,9(7,1-12,7)$ \\
\hline Familiar ou conhecida com câncer de mama & 17 & $4,3(2,3-6,3)$ & 10 & $2,3(0,8-3,6)$ \\
\hline Medo de ter câncer & 3 & $0,7(0-1,6)$ & 13 & $2,9(1,3-4,5)$ \\
\hline Mulher acima de 40 anos deve fazer & 17 & $4,3(2,3-6,3)$ & 25 & $5,6(3,4-7,8)$ \\
\hline Acompanhamento/rotina & 297 & $75,4(71,1-79,6)$ & 352 & $79,3(75,5-83,1)$ \\
\hline Total & 394 & 100,0 & 444 & 100,0 \\
\hline \multicolumn{5}{|l|}{ Motivos para não fazer a mamografia } \\
\hline Medo/vergonha & 13 & $30,2(15,9-44,5)$ & 2 & $5,3(0-12,7)$ \\
\hline Acha desnecessário & 8 & $18,6(6,5-30,7)$ & 16 & $42,1(25,7-58,6)$ \\
\hline Falta de solicitação & 22 & $51,2(35,6-66,7)$ & 20 & $52,6(36,0-69,3)$ \\
\hline Total & 43 & 100,0 & 38 & 100,0 \\
\hline
\end{tabular}

clínico, e para as mulheres entre 50 e 69 anos, o percentual foi $56,8 \% 32$. Apesar das diferenças metodológicas, os estudos demonstraram que a população feminina encontra barreiras para realização de forma adequada do controle do câncer de mama.

Na cidade de Maringá, Paraná, um inquérito de base populacional, com mulheres de 40 a 69 anos, demonstrou que $84,4 \%$ haviam realizado a última mamografia em um intervalo de tempo menor de dois anos. Dentre estas, as que apresentaram menor prevalência de realização do exame foram aquelas com idade entre 51 a 60 anos 33 .

Neste estudo, a menor prevalência de realização do exame foi encontrada em mulheres de 60 a 69 anos. De acordo com a literatura 25,34, estas são as mulheres com as maiores taxas de incidência de câncer de mama. Em Goiás, ao analisar os exames de mamografia realizados no estado no ano de 2009, encontrou-se maior taxa de realização nas mulheres 40 a 49 anos comparadas às de 60 a 69 anos 35. Estas mulheres, de 60 a 69 anos, podem estar deixando de fazer a mamografia, e consequentemente estarem em maior risco de diagnóstico em estádios avançados.

Idosas de baixa escolaridade e baixa renda tiveram menores prevalências de realização anual do exame. As mulheres com baixa escolaridade são aquelas com menor chance de realização de mamografia 16,36 , assim como aquelas com menor renda 16,36,37. Esta associação com escolaridade e renda também foi encontrada em serviços públicos de saúde, nos quais a prática adequada foi associada à alta escolaridade e maior renda 20 . Além disto, mulheres de alta escolaridade são as que possuem as maiores taxas de diagnóstico de câncer de mama, e aquelas de baixa renda apresentam duas vezes mais chance de serem diagnosticadas em estágio avançado da doença que mulheres de alta renda 38 . Segundo Oliveira et al. 16, historicamente, mulheres de maior renda possuem maior acesso ao exame, entretanto o crescimento da economia pode impulsionar mulheres de baixa renda a terem mais acesso ao exame.

Além disto, mulheres com maior nível de escolaridade 18 e maior renda 20,21 seguem melhor as recomendações de realização do exame. Possivelmente estas mulheres possuem mais acesso à informação e recursos de saúde, o que possibilita a realização dos exames na periodicidade estabelecida.

Estes achados apontam para uma iniquidade em saúde. Uma das maneiras de garantir acesso igualitário é melhorar a comunicação em saúde e a eficácia da informação transmitida. A comunicação determina a dinâmica da estrutura so- 
cioeconômica e sociocultural e sua relação com o sistema de saúde. Além disto, o papel da comunicação efetiva auxilia no acesso aos serviços de saúde para todos os indivíduos, independente da classe social ou escolaridade 39 . Da mesma forma, deve-se promover equidade no acesso aos serviços de saúde, independente de nível socioeconômico, para que todas as mulheres tenham acesso indistinto a atendimento médico e a exames preventivos.

No presente estudo, as mulheres idosas que possuíam companheiro apresentaram maior prevalência de realização anual do exame. Novaes \& Mattos 19 encontraram a associação de não realização de mamografia por mulheres idosas sem companheiro. No Brasil, as mulheres com companheiro possuem maior chance de realizar mamografia do que as demais 16,20. Entretanto, nos Estados Unidos, o estado civil não influenciou nas taxas de diagnóstico do câncer de mama 38. Amorim et al. 17 levantam a hipótese de que mulheres sem companheiros demandam menos atenção nos serviços de saúde, e realizam menor quantidade de exames preventivos, por acharem que não há necessidade de frequentar consultas ginecológicas, por estarem sexualmente inativas. Em especial para mulheres idosas esta hipótese pode ser verdadeira, pois estas não recorrem a métodos contraceptivos, devido à menopausa, e deixam de frequentar aqueles serviços. Entretanto, com o aumento da expectativa de vida, a busca pelos serviços de saúde pela população idosa deve aumentar e os serviços devem estar preparados para o atendimento integral, inclusive relacionado ao câncer de mama 17 .

Na presente pesquisa, a associação entre a realização de mamografia e possuir planos de saúde privado deu-se somente em mulheres adultas. Aquelas que possuíam plano de saúde tiveram prevalência de realização anual do exame $40 \%$ maior em relação as que não possuem, mesmo após ajuste por idade. No Brasil, no período compreendido entre 2003 e 2008, é percebida melhora de acesso e utilização da mamografia independentemente da vinculação a um plano de saúde, mas para as que possuem, dobram as chances de realização do exame 16. Os dados de VIGITEL de 2008 demonstram que as beneficiárias de planos de saúde, de 50 a 69 anos, apresentam prevalência $49 \%$ maior de realização de mamografia em um período de dois anos comparadas as não beneficiárias, ajustado por idade e escolaridade ${ }^{40}$. Estudo realizado na cidade de Taubaté, São Paulo, mostrou que 84,2\% das mulheres que haviam realizado rastreamento mamográfico nos dois anos anteriores à pesquisa tinham cobertura de planos de saúde pri- vados, e que estas tinham recebido orientações sobre a importância do exame 23 .

A associação da realização anual da mamografia à posse de plano de saúde pode estar relacionada ao fato de médicos do sistema privado seguirem as recomendações de rastreamento anual, o que ocasiona que mulheres atendidas por médicos de planos privados realizem mais exames que as atendidas pelo Sistema Único de Saúde (SUS). Amorim et al. 17 reforçam esta hipótese ao afirmar que a restrição dada pelas recomendações do INCA em rastrear apenas mulheres de 50 a 69 anos, faz com que solicitações de mamografias realizadas em serviços não vinculados ao SUS tenham maior flexibilidade para indicação e realização dos exames.

A realização da mamografia no Brasil é associada a frequentar consultas médicas: mulheres que não frequentaram consultas médicas nos últimos 12 meses possuem um terço da chance de realização do exame do que aquelas que frequentaram 16. Assim, as mulheres adultas com vinculação a plano de saúde podem ter frequentado maior número de consultas médicas, variável que não foi abordada no estudo, e ter contribuído para a diferença de prevalência encontrada.

Neste estudo cerca de $10 \%$ das adultas e $9 \%$ das idosas nunca haviam realizado o exame. Os percentuais encontrados diferem muito do $\mathrm{Mu}$ nicípio de Campinas em que 42,5\% das mulheres acima de 40 anos nunca haviam realizado o exame 17. Marchi \& Gurgel 23 referem que a grande proporção de mulheres que não realizam o exame são as que não possuem plano de saúde privado, em virtude de maior dificuldade de acesso aos serviços de saúde. Os achados demonstram que as mulheres de Florianópolis realizam o exame, mesmo entre aquelas de 40 a 49 anos, em relação às quais as recomendações do Ministério de Saúde ${ }^{9}$ são somente para rastreamento por exame clínico.

Entre as barreiras estudadas estão a falta de solicitação médica, medo ou vergonha do exame e achar desnecessário. A falta de solicitação médica foi o motivo mais relatado pelas mulheres, tanto adultas quanto idosas. No estudo de Marinho et al. 20 mais de $80 \%$ das entrevistadas referiram esta como a principal barreira. No Brasil, além do médico, os enfermeiros também podem solicitar a mamografia de rastreamento 41 , e com isto, aumentar a adesão. Entretanto, a falta de solicitação do exame pode ser um dos problemas do rastreamento oportunístico, cuja realização necessita da solicitação da busca da mulher pelos serviços. O medo referente ao exame pode estar tanto relacionado a um diagnóstico do câncer de mama, quanto ao exame ser doloroso, motivos que também são conside- 
rados barreiras para a sua realização 42 . E quanto ao achar desnecessário mostra principalmente a falta de conhecimento sobre a detecção precoce do câncer de mama 43 .

Segundo a literatura 18,42, o diálogo pode ajudar a vencer dificuldades, como a falta de solicitação médica, aliviando possíveis medos e ansiedades e informando sobre a importância da realização do exame, e consequentemente aumentando a adesão ao exame.

Este estudo apresentou limitações. Uma delas é o fato de as informações necessárias para determinar a realização de mamografia, o intervalo e tempo decorrido do último exame, terem sido obtidas por entrevista. Neste caso pode ter ocorrido viés de memória, não se lembrar sobre o tempo decorrida da realização do último exame e informar o tempo que considera adequado, o que levaria ao aumento da realização anual. Além disto, a análise não foi ajustada para a informação se a mulher tinha parentes ou amigas com história de câncer de mama, pois esta informação não estava disponível, e pode aumentar as chances de participarem mais frequentemente de exames de rastreamento.
Em conclusão, o artigo abordou a realização anual de mamografia de acordo com as recomendações de sociedade científica, a American Cancer Society ${ }^{8}$, e profissionais, a Sociedade Brasileira de Mastologia 14 e o Colégio Brasileiro de Radiologia e Diagnóstico por Imagem 15. Esta é influenciada por fatores socioeconômicos, diferentes em adultas e idosas: entre as primeiras, possuir plano de saúde privado, e para as últimas, ter mais de cinco anos de estudo, pertencer ao quartil mais alto de renda e ter companheiro entre as idosas.

A informação sobre a realização de mamografia deve ser disseminada entre todas as mulheres, independente de condicionantes sociais e econômicos, promovendo a equidade de utilização dos exames preventivos. Uma estratégia que pode ser utilizada para promover a equidade é a mobilização social, com a participação de governo, grupos de pacientes e comunidade, que podem auxiliar no aumento da adesão ao rastreamento mamográfico, e a redução da mortalidade por câncer de mama. Além disto, o programa de controle e da qualidade da mamografia deve ser implantada em todos os serviços para que os exames apresentem padrão para o diagnóstico do câncer de mama.

\section{Resumen}

El objetivo fue identificar los factores asociados con la realización de la mamografía anual en mujeres de 40 a 69 años que viven en Florianópolis, Santa Catarina, Brasil. Los datos fueron obtenidos en dos encuestas de población, una con y otra con los adultos mayores en 2009-2010. Se estimó la prevalencia de la realización de la mamografía anual, y regresión empleada univariante y multivariante de Poisson, para identificar los factores asociados. El estudio incluyó a 447 adultos y 510 ancianos. La prevalencia anual de logros entre los adultos fue de un 43,5\% (IC95\%: 38,8-48,2), y quienes cuentan con planes de salud privados incrementaron esta prevalencia. En el caso de las personas mayores, un 38,3\% (IC95\%: 34,0-42,6) se sometió a una mamografía anual y factores asociados como: contar con más de cinco años de estudio o pertenecer al mayor cuartil de ingreso. El uso de la mamografía debe ser independiente de cualquier condición social, de cualquier edad, promoviendo así la reducción de la mortalidad por cáncer de mama.

Diagnóstico Precoz; Mamografía; Neoplasias de la Mama; Encuestas Epidemiológicas 


\section{Colaboradores}

I. J. C. Schneider e E. d'Orsi participaram da concepção e desenho do estudo, organização do trabalho e condução da análise estatística, interpretação dos resultados, elaboração e revisão do manuscrito. M. W. C. Giehl colaborou na concepção e desenho do estudo, organização do trabalho, condução da análise estatística, revisão do manuscrito. A. F Boing contribuiu na concepção e desenho do estudo e revisão do manuscrito.

\section{Referências}

1. International Agency for Research on Cancer. Breast cancer screening. Lyon: International Agency for Research on Cancer; 2002.

2. Godinho ER, Koch HA. O perfil da mulher que se submete a mamografia em Goiânia: uma contribuição a "Bases para um programa de detecção precoce do câncer de mama”. Radiol Bras 2002; 35:139-45.

3. American Cancer Society. Cancer facts \& figures 2009. Atlanta: American Cancer Society; 2009.

4. World Health Organization. Early detection. Cancer control: knowledge into action. WHO guide for effective programmes; module 3. Geneva: World Health Organization; 2007.

5. Nelson HD, Tyne K, Naik A, Bougatsos C, Chan BK, Humphrey L. Screening for breast cancer: an update for the U.S. Preventive Services Task Force. Ann Intern Med 2009; 151:727-37.

6. Mandelblatt JS, Cronin KA, Bailey S, Berry DA, Koning HJ, Draisma G, et al. Effects of mammography screening under different screening schedules: model estimates of potential benefits and harms. Ann Intern Med 2009; 151:738-47.

7. American Cancer Society (ACS). Breast cancer facts \& figures 2009-2010. Atlanta: American Cancer Society: 2010.

8. Smith RA, Cokkinides V, Brooks D, Saslow D, Brawley OW. Cancer screening in the United States, 2010: a review of current American Cancer Society guidelines and issues in cancer screening. CA Cancer J Clin 2010; 60:99-119.

\section{Agradecimentos}

Agradecemos aos técnicos do Instituto Brasileiro de Geografia e Estatística (IBGE) e da Secretaria Municipal de Saúde de Florianópolis pelo auxílio na operacionalização destes estudos. Ao CNPq pelo financiamento.
9. Instituto Nacional de Câncer. Controle do câncer de mama: documento de consenso. Rio de Janeiro: Instituto Nacional de Câncer; 2004.

10. Instituto Nacional de Câncer. Mamografia: da prática ao controle. Rio de Janeiro: Instituto Nacional de Câncer; 2007.

11. Tiezzi DG. Cancer de mama: um futuro desafio para o sistema de saúde nos países em desenvolvimento. Rev Bras Ginecol Obstet 2010; 32:257-9.

12. Smith RA. International programs for the detection of breast cancer. Salud Pública Méx 2011; 53:394-404.

13. Miles A, Cockburn J, Smith RA, Wardle J. A perspective from countries using organized screening programs. Cancer 2004; 101(5 Suppl):1201-13.

14. Sociedade Brasileira de Mastologia. Recomendações da X Reunião Nacional de Consenso da Sociedade Brasileira de Mastologia, rastreamento do câncer de mama na mulher brasileira. http://www. sbmastologia.com.br/downloads/reuniao_de_ consenso_2008.pdf (acessado em 06/Abr/2013).

15. Colégio Brasileiro de Radiologia e Diagnóstico por Imagem. Mamografia salva vidas. Boletim CBR 2010; (264):18-21.

16. Oliveira EXG, Pinheiro RS, Melo ECP, Carvalho MS. Condicionantes socioeconômicos e geográficos do acesso à mamografia no Brasil, 2003-2008. Ciênc Saúde Coletiva 2011; 16:3649-64. 
17. Amorim VMSL, Barros MBA, César CLG, Carandina L, Goldbaum M. Fatores associados a não realização da mamografia e do exame clínico das mamas: um estudo de base populacional em Campinas, São Paulo, Brasil. Cad Saúde Pública 2008; 24:2623-32.

18. Molina L, Dalben I, De Luca LA. Análise das oportunidades de diagnóstico precoce para as neoplasias malignas de mama. Rev Assoc Méd Bras 2003; 49:185-90.

19. Novaes CO, Mattos IE. Prevalência e fatores associados a não utilização de mamografia em mulheres idosas. Cad Saúde Pública 2009; 25 Suppl 2:S310-20

20. Marinho LAB, Cecatti JG, Osis MJ, Gurgel MS. Knowledge, attitude and practice of mammography among women users of public health services. Rev Saúde Pública 2008; 42:200-7.

21. Marinho LAB. Conhecimento, atitude e prática do auto-exame da mama e do exame de mamografia em usuárias dos centros de saúde do município de Campinas [Tese de Doutorado]. Campinas: Universidade Estadual de Campinas; 2001.

22. Sclowitz ML, Menezes AMB, Gigante DP, Tessaro S. Condutas na prevenção secundária do câncer de mama e fatores associados. Rev Saúde Pública 2005; 39:340-9.

23. Marchi AA, Gurgel MSC. Adesão ao rastreamento mamográfico oportunístico em serviços de saúde públicos e privados. Rev Bras Ginecol Obstet 2010; 32:191-7.

24. Marchi AA, Gurgel MSC, Fonsechi-Carvasan GA. Rastreamento mamográfico do câncer de mama em serviços de saúde públicos e privados. Rev Bras Ginecol Obstet 2006; 28:214-9.

25. Instituto Nacional de Câncer José Alencar Gomes da Silva. Perfil da morbimortalidade brasileira do câncer de mama. Rio de Janeiro: Instituto Nacional de Câncer José Alencar Gomes da Silva; 2012.

26. De Castro Mattos JS, Mauad EC, Syrjänen K, Longatto-Filho A, Haikel RL, Da Costa et al. The impact of breast cancer screening among younger women in the Barretos Region, Brazil. Anticancer Res 2013; 33:2651-5.

27. Freitas-Junior R, Freitas NMA, Curado MP, Martins E, Silva CMB, Raha RMS, et al. Incidence trend for breast cancer among young women in Goiânia, Brazil. São Paulo Med J 2010; 128:81-4.

28. Dupont WD. Statistical modeling for biomedical researchers: a simple introduction to the analysis. 2nd Ed. Cambridge: Cambridge University Press; 2009.

29. Sadler GR, Ko CM, Cohn JA, White M, Weldon RN, Wu P. Breast cancer knowledge, attitudes, and screening behaviors among African American women: the Black cosmetologists promoting health program. BMC Public Health 2007; 7:57.

30. Segri NJ, Francisco PMSB, Alves MCGP, Barros MBA, Cesar CLG, Goldbaum M, et al. Práticas preventivas de detecção de câncer em mulheres: comparação das estimativas dos inquéritos de saúde (ISA-Capital) e vigilância de fatores de risco e proteção para doenças crônicas por inquérito telefônico (VIGITEL-São Paulo). Rev Bras Epidemiol 2011; 14 Suppl 1:31-43.
31. Moreira CB, Bezerra KC, Mendes IC, Santos MCL, Oriá MOB, Fernandes AFC. Prevalência do exame mamográfico em mulheres brasileiras no período de 2009 a 2010. Rev Enferm UERJ 2013; 21:151-5.

32. Barreto ASB, Mendes MFM, Thuler LCS. Avaliação de uma estratégia para ampliar a adesão ao rastreamento do câncer de mama no Nordeste brasileiro. Rev Bras Ginecol Obstet 2012; 34:86-91.

33. Matos JC, Pelloso SM, Carvalho MDB. Fatores associados à realização da prevenção secundária do câncer de mama no Município de Maringá, Paraná, Brasil. Cad Saúde Pública 2011; 27:888-98.

34. Ferlay J, Shin HR, Bray F, Forman D, Mathers C, Parkin DM. GLOBOCAN 2008: cancer incidence and mortality worldwide. Lyon: International Agency for Research on Cancer; 2010.

35. Corrêa RS, Freitas-Júnior R, Peixoto JE, Rodrigues DCN, Lemos MEF, Marins LAP, et al. Estimativas da cobertura mamográfica no Estado de Goiás, Brasil. Cad Saúde Pública 2011; 27:1757-67.

36. Samah AA, Ahmadian M. Socio-demographic correlates of participation in mammography: a survey among women aged between 35-69 in Tehran, Iran. Asian Pac J Cancer Prev 2012; 13:2717-20.

37. Zhao DH, Zhang ZR, Rao KQ. Health insurance and household income associated with mammography utilization among American women, 20002008 Chin Med J (Engl) 2011; 124:3320-6.

38. Clegg LX, Reichman ME, Miller BA, Hankey BF, Singh GK, Lin YD, et al. Impact of socioeconomic status on cancer incidence and stage at diagnosis: selected findings from the surveillance, epidemiology, and end results: National Longitudinal Mortality Study. Cancer Causes Control 2009; 20:417-35.

39. Thiede M, McIntyre D. Information, communication and equitable access to health care: a conceptual note. Cad Saúde Pública 2008; 24:1168-73.

40. Malta DC, Moura EC, Oliveira M, Santos FP. Usuários de planos de saúde: morbidade referida e uso de exames preventivos, por inquérito telefônico, Brasil, 2008. Cad Saúde Pública 2011; 27:57-66.

41. Instituto Nacional de Câncer. Solicitação de mamografia de rastreamento por enfermeiros. http:// www2.inca.gov.br/wps/wcm/connect/d8f78f004e b6845189c09bf11fae00ee/nota_tecnica_solicitacao _mamografia_pelo_enfermeiro.pdf?MOD=AJPE RES\&CACHEID $=$ d8f78f004eb6845189c09bf $11 \mathrm{fae}$ 00ee (acessado em 20/Jan/2014).

42. Dundar PE, Ozyurt BC, Erdurak K. Sociodemographic determinants of nonattendance in a Population-Based Mammography Screening Program in the city of Manisa, Turkey. ScientificWorldJournal 2012; 2012:816903.

43. Akhigbe AO, Omuemu VO. Knowledge, attitudes and practice of breast cancer screening among female health workers in a Nigerian urban city. BMC Cancer 2009; 9:203.

Recebido em 12/Set/2013

Versão final reapresentada em 05/Fev/2014

Aprovado em 17/Fev/2014 